\title{
Comparitive Study of Hysteroscopy and Transvaginal Sonography in Screening of Patients with Abnormal Uterine Bleeding
}

\author{
Dr.N.Kurinjipriya M.D ${ }^{1,}$ Dr.Teresa Karpaga Selvi M.D \\ Department of Obsterics and Gynaecology Coimbatore Medical College Hospital Coimbatore, Tamilnadu
}

\begin{abstract}
Aims And Objectives:
To Study The Efficacy Of Hysteroscopy As A Screening Method In Patients With Abnormal Uterine Bleeding.

To Compare The Usefulness Of Hysteroscopy And Transvaginal Ultrasound In Abnormal Uterine Bleeding.

To Correlate The Results Of Hysteroscopy And Tvs With Histopathological Examination.
\end{abstract}

\section{Patient Population}

\section{Materials And Methods}

Our study includes 50 women of age varying between 28 to 55 years who were admitted. We included only the patients with technical success in transvaginal sonography and Hysteroscopy in this study. Finally 50 patients with pathological confirmation were included in this study. All 50 patients were first evaluated with transvaginal sonography followed by Hysteroscopy and traditional curettage with cervical biopsy performed after 2 days following transvaginal sonography[7MHz]The pathological findings are then correlated with ultrasound finding and diagnosis by hysteroscopy.

\section{Criteria for Selection}

1. Patients who had AUB for more than 6 months were selected

2. Patients in Reproductive and perimenopausal age were selected

3. Both Nulliparous and parous women were included

4. Patients in pubertal age group were excluded

5. Both treated and untreated patients were taken

6. Patients with any temporary method of contraception for the past 6 months were excluded

7. Patients who underwent abortion and pregnant were excluded

8. Patients with any other medical or surgical illness were excluded

9. Lactating mothers were excluded

10. Informed consent from the patients taken. Patients were also investigated if they have Intra-uterine device or not, oral contraceptive use, regular drug or hormonal preparation usage. Former gynaecological operations and applications recorded. .Cervix,cervical canal, myometrium and ovaries were examined in sagittal and coronal section. After inspection of morphological pattern of endometrium, endometrial thickness of external borders measured and recorded in thickest place in longitudinal plane.

\section{Review Of Literature}

\section{Diagnostic Hysteroscopy \\ Instrumentation : \\ Telescopes}

The most important piece of equipment for hysteroscopy is the lens or telescope. The optics as well as the fiberoptic illumination bundles are packaged together in this single instrument. Most rigid telescopes range in diameter from 2 to $4 \mathrm{~mm}$ (o.d.). The best light shower and optical resolution are likely to be found in $4 \mathrm{~mm}$ (o.d.) instruments. However, for office hysteroscopy, as well as operative procedures, a $3 \mathrm{~mm}$ telescope can produce an acceptable video image particularly when magnified by means of a zoom video camera.

- The magnifying eyepiece.

- The transmitting lens systems.

- Objective lens

\section{Fiberoptic Light Cable}

Fiberoptic cables transmit intense cold light to the telescope and form a conduit, which connects the high intensity (heat producing) generator to the telescope. The cable is filled with many incoherent drawn-out 
glass fibers capable of conducting light from the generator to the terminus of the cable.Inspection of the periphery of the cable in a darkened room can also reveal fibre disruption. This appears as light transmission through the sides of the cable.

\title{
Hysteroscopic Sheaths
}

Two general categories of sheaths are used for hysteroscopic procedures : diagnostic and operating. A sheath is required for panoramic hysteroscopy in order to serve as a conduit through which to instill the distending medium into the uterine cavity. The diagnostic sheath fulfils this singular requirement and measures approximately $4-5 \mathrm{~m}$ in outer diameter (when coupled to a 3-4 $\mathrm{mm}$ telescope).

\section{Abnormal Uterine Bleeding}

Abnormal uterine blooding is the most common complaint of patients consulting the gynaecologist and provides the most frequent indication for hysterectomy. Dilatation and Curettage has been the diagnostic method of choice for many decades for these cases.

However, for diagnosing, non-malignant intrauterine pathology such as endometrial polyps, and intrauterine leiomyoms which may cause uterine bleeding disorders, D and C appears to be unreliable (Wamstekar, 1977, 1948 a; Gimpelson and Rappold. 1988, Loffer 1989; Motashaw and Dave. 1990).

Direct hysteroscopic inspection with adequate distention and visualization discloses almost every intrauterine abnormality with high accuracy. Additionally, it enables exact localization of the pathology and determination of its intracavitary extent, However, for the diagnosis of endometritis and adenomyosis, conclusive hysteroscopic criteria are still lacking.

For histologic examination selective samples of any abnormal tissue can be obtained by visually controlled biopsies. A significant percentage of benign intrauterine pathology disclosed by hysteroscopic diagnosis in patients with abnormal uterine bleeding can be treated with minimally invasive transcervical hysteroscopic endosurgery.

As the majority of intrauterine disorders resulting in abnormal uterine bleeding in the reproductive phase of life, the climacteric and the postmenopausal period are benign types of pathology, D and C can be longer hold its position as the primary diagnostic method for patients with abnormal uterine bleeding. Today ambulant or outpatient hysteroscopy with visually directed biopsies or directed curettage is to be recommended as the diagnostic method of choice. Although D and $\mathrm{C}$ will seldom fail to disclose endometrial cancer and hyperplasia, hysteroscopic investigation additionally enables the early detection of small endometrial cancers and determination of the localization, size and extent of the neoplasia and / or its precursors. Notwithstanding the above - mentioned considerations it seems to be sensible to recommend reducing transabdominal spill of distention medium as much as possible in these cases. This can be achieved reducing the intrauterine working pressure during hysteroscopy in cases suspected of endometrical cancer.

\author{
Specific indications for hysteroscopic diagnosis in patients with abnormal uterine bleeding are : \\ - Menorrhagia. \\ - Metrorrhagia \\ - Intermenstrual bleeding \\ - Postmenopausal bleeding \\ - Intrauterine or endometrial abnormality on TVS or HSG
}

Patients admitted, evaluated to rule out systemic illness and then subjected to hysteroscopy after informed consent. Under strict aseptic precautions, under Total intra-venous Anaesthesaia, patient put in Lithotomy position KARAL STORZ hysteroscope introduced. Cervical canal examined followed by a panoramic inspection of while endometrial cavity in relation to uterine landmarks - cornea, tubal ostia and fundus.

Endometrial tissue covering smooth surface - pedunculated or no - pendunculated structure are evaluated as polyp. Lesions not concerned with endometrium, shiny as pearl, sessile appearing and vascularisation are submucous myomas.

If endometrial surface is smooth or thickened in polypoidal appearance, when pressed with hysteroscope, endometrial groove is seen it is called Hyperplasia. When this thickening is together with high grade irregularity, it is called Atypical Hyperplasia. If necrotic areas, glandular and vascular disorganization is present in addition to this appearance endometrial carcinoma is thought of.

Dark red coloured relatively with smooth surface or lesions seen independent from necrotic endometrium are evaluated as retention of placental tissue. All other finding except from above are accepted as normal endometrium. 
Comparitive Study Of Hysteroscopy And Transvaginal Sonography In Screening Of Patients...

Normal endometrium and atrophic endometrium are accepted as normal hysteroscopic findings. Dilatation and curettage is performed to all patients. Obtained material is preserved in formalin and set to Histopathology laboratory for confirmation of diagnosis. Endometrial hyperplasia, polyp, myoma and placental polyps are accepted as pathological results.

When no material is obtained and histological finding as secretory endometrium, proliferative endometrium, normal and Atrophic endometrium are evaluated. Chi square and Fischer extract tests are used to compare the rates $\mathrm{P}$ value $<0.05$ as accepted as significant.

\section{Analysis Of The Study}

Patients who have undergone sterilisation had increased incidence of AUB. Probable reason may beThe blood supply to the ovary is altered and The axis of ovary is sometimes changed due to adhesions. .56 \% of patients in reproductive age group had ovulatory AUB . $20 \%$ of patients among perimenopausal age group had anovulatory AUB.

Table 1 Tvs Endometrial Thickness

\begin{tabular}{|c|c|c|c|c|c|c|c|c|c|c|c|c|}
\hline & \multicolumn{12}{|c|}{ Endometrial Thickeness } \\
\hline & \multicolumn{2}{|c|}{$\begin{array}{c}1 \\
\mathrm{Mm} \\
\end{array}$} & \multicolumn{2}{|c|}{$1-3 \mathrm{~mm}$} & \multicolumn{2}{|c|}{$3-5 \mathrm{~mm}$} & \multicolumn{2}{|c|}{$5-8 \mathrm{~mm}$} & \multicolumn{2}{|c|}{$8-10 \mathrm{~mm}$} & \multicolumn{2}{|c|}{$\begin{array}{l}>1 \\
\mathrm{~cm}\end{array}$} \\
\hline & No & $\%$ & No & $\%$ & No & $\%$ & No & $\%$ & No & $\%$ & No & $\%$ \\
\hline $\begin{array}{l}\text { Reproductive } \\
\text { Age group }\end{array}$ & 0 & 0 & 15 & 30 & 19 & 38 & 1 & 2 & 0 & 0 & 0 & 0 \\
\hline $\begin{array}{l}\text { Perimenopausal } \\
\text { Age group }\end{array}$ & 0 & 0 & 5 & 10 & 1 & 2 & 5 & 10 & 3 & 6 & 1 & 2 \\
\hline
\end{tabular}

In the study of the endometrial thickeness of the reproduction age group, pencil line endometrium was nil, 15 had 1-3 mm, 19 patients 3-5mm thickeness, 1 had 5-8 mm thickness, none had $>1 \mathrm{~cm}$ thicknessAmong perimenopausal age group, pencil line endometrium was nil, 5 had 1-3 mm thickness, patient had 3-5 mm thickness and 3 patients had $8-10 \mathrm{~mm}$ thickness and 1 patient had thickness $>1 \mathrm{~cm}$.

Table 2

\begin{tabular}{|c|c|c|c|c|c|c|c|c|c|c|c|c|c|c|c|c|}
\hline \multirow[t]{2}{*}{ Table -2} & \multicolumn{2}{|c|}{ Atropic } & \multicolumn{2}{|c|}{$\begin{array}{l}\text { Proliferativ } \\
\mathrm{e}\end{array}$} & \multicolumn{2}{|c|}{ Secretory } & \multicolumn{2}{|c|}{$\begin{array}{l}\text { Adenomat } \\
\text { ous }\end{array}$} & \multicolumn{2}{|c|}{ Atypical } & \multicolumn{2}{|c|}{ AdenoCa } & \multicolumn{2}{|c|}{ Endometritis } & \multicolumn{2}{|c|}{$\begin{array}{c}\text { Cystogland } \\
\text { ular } \\
\text { hyperplasia }\end{array}$} \\
\hline & No & $\%$ & No & $\%$ & No & $\%$ & No & $\%$ & No & $\%$ & No & $\%$ & No & $\%$ & No & $\%$ \\
\hline $\begin{array}{l}\text { Reproducti } \\
\text { ve } \\
\text { Age group }\end{array}$ & 0 & 0 & 16 & 32 & 11 & 22 & 1 & 2 & 0 & 0 & 0 & 0 & 1 & 2 & 6 & 12 \\
\hline $\begin{array}{l}\text { Perimenopa } \\
\text { usal } \\
\text { Age group }\end{array}$ & 0 & 0 & 5 & 10 & 6 & 12 & 0 & 0 & 0 & 0 & 0 & 0 & 0 & 0 & 4 & 8 \\
\hline
\end{tabular}

In the reproductive age group HPE consisted mostly of proliferative (32\%) and secretory (22\%) pattern . One case of Adenomatous pattern and one case of endometritis detected.12\% cases of cystoglandular hyperplasia were noted.

In the perimenopausal age group, $12 \%$ of secretory changes, $10 \%$ with proliferative changes and $8 \%$ of cases with cystoglandular hyperplasia were noted.

Table 3

Distribution of uterine pathology regarding TVS

\begin{tabular}{|l|l|l|}
\hline Urerine Pathology & No. Of cases & $\%$ \\
\hline Hyperplasia & 9 & 18 \\
\hline Polyp & 5 & 10 \\
\hline Myoma & 2 & 4 \\
\hline Polyp + Myoma & 0 & 0 \\
\hline Cancer & 0 & 0 \\
\hline No Pathology & 34 & 38 \\
\hline
\end{tabular}

In both reproductive and perimenopausal age group, according to transvaginal sonography, 9 cases showed endometrial hyperplasia, 5 cases were with endometrial polyp, 2 cases showed submucous myoma and the remaining 34 cases were with nil pathology. 
Comparitive Study Of Hysteroscopy And Transvaginal Sonography In Screening Of Patients...

Table -4 Distribution of uterine pathology regarding hysteroscopy

\begin{tabular}{|c|c|c|}
\hline Urerine Pathology & No. Of cases & $\%$ \\
\hline Hyperplasia & 1.5 & 30 \\
\hline Polyp & 12 & 34 \\
\hline Myoma & 2 & 4 \\
\hline Polyp + Myoma & 1 & 2 \\
\hline Cancer & 0 & 0 \\
\hline No Pathology & 20 & 40 \\
\hline
\end{tabular}

According to hysteroscopy, 15 cases were diagnosed with endometrial hyperplasia, 12 cases showed endometrial polypoidal growths, 2 cases were diagnosed as submucous myoma, 1 case as with both endometrial polyp and submucous myoma and 20 Cases showed no pathology.

Table 5 Distribution of uterine pathology regarding D \& C

\begin{tabular}{|l|l|l|}
\hline Urerine Pathology & No. Of cases & $\%$ \\
\hline Hyperplasia & 10 & 20 \\
\hline Polyp & 5 & 10 \\
\hline Myoma & 0 & 0 \\
\hline Polyp + Myoma & 0 & 0 \\
\hline Cancer & 0 & 0 \\
\hline Adenomatous & 1 & 2 \\
\hline Endometritis & 1 & 2 \\
\hline No pathology & 33 & 66 \\
\hline
\end{tabular}

According to dilatation and curettage biopsy, 10 cases (20\%) showed cystoglandular hyperplasia, 5 cases (10\%) showed polypoidal growth, 1 case (2\%) of endometritis and 33 cases $(66 \%)$ were with normal histopathology.

Table 6 -Distribution of Intra uterine pathology according to TVS, D\&C

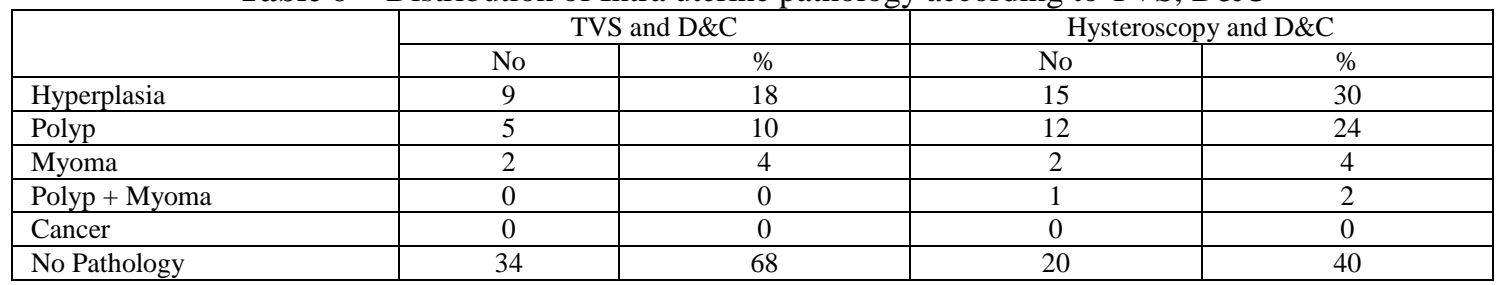

\section{Discussion}

Abnormal uterine bleeding is a common problem (Couher) and is the main indication for hysteroscopic evaluation. Hysteroscopy and USG are now being increasingly used not only for detecting functional disorders of endometrium, but also for excluding various unsuspected organic diseases of the endometrium like cancer and tuberculosis.

In this series 50 patients with AUB were taken for study. For all 50 patients, TVS, hysteroscopy and $\mathrm{D} \& \mathrm{C}$ were done in the premenstrual phase and analysis of the study had been done. The study period consisted of 2 years - during the year 2013-2015 at Coimbatore medical college hospital.

In all the patients, endometrial thickness measured by Sonogram was compared with HPE.

> Comparing the distribution and diagnosis of intra uterine pathology according to transvaginal sonography, D\&C and hysteroscopy showed that $30 \%$ hyperplasia was detected by combination of hysteroscopy and D\&C whereas TVS and D\&C could detect only $18 \%$ of hyperplasia.

$>$ Combination of hysteroscopy and D\&C could detect $24 \%$ of cases with endometrial polyp while TVS and $\mathrm{D} \& \mathrm{C}$ could detect only $10 \%$ of cases with endometrial polyp.

$>$ Both combination of transvaginal sonography and D\&C could detect $4 \%$ of cases as with sub mucous myoma but combination hysteroscopy and $\mathrm{D} \& \mathrm{C}$ in addition, could detect $2 \%$ of case as with both myoma and polyp.

$>40 \%$ of cases showed no pathology according to diagnostic hysteroscopy and D\&C whereas TVS and D\&C could not detect any pathology in $68 \%$ of AUB cases.

$>$ Thus combination of diagnostic hysteroscopy and D\&C has better diagnostic value when compared with transvaginal sonography and D\&C. 


\section{Summary}

Other A total of 50 cases of DUB have been selected. 35 from reproductive age group and 15 from perimenopausal age group. These patients were subjected to TVS, hysteroscopy and D\&C on two successive days in the premenstrual phase. diseases like bleeding disorders were excluded. TVS, diagnostic hysteroscopy and $\mathrm{D} \& \mathrm{C}$ were done in premenstrual phase. There was an excellent correlation of endometrial thickness with that of HPE of endometrium. Most patients with 3-5mm thickness were of secretory and proliferative pattern.

There were no complications encountered during the above said procedures. Blood group of B was an incidental finding and there is no literature of support a particular group in AUB.

Most of the endometrial hyperplasia, myomas and polyps were diagnosed by diagnostic hysteroscopy when compared to TVS or D\&C. Diagnostic hysteroscopy can be done in an office setting as a day care procedure and is considered as a safe procedures with minimal complications. Women with menorrhagia under 40 years may require hysteroscopic investigation if intra uterine pathology is suspected following transvaginal ultrasound scan or due to severity and persistence of symptoms despite recommended medical treatment (RCOG) Hysteroscopy combined with histological examination is considered as "Gold standard" for evaluating a case with abnormal uterine bleeding. (Widrich et al, Bakour).

\section{Conclusion}

Abnormal uterine bleeding (AUB) is one of the most commonly encountered condition in OPD. The most probable cause of abnormal uterine bleeding depends on the patients reproductive age and the likelihood of endometrial pathology.

Achary V, Mehte S and Rander A is March 2003, studied 100 patients and conluded that for detecting submucous myomas and endometrial polyp, hysteroscopy has 100 percent sensitivity, specificity and very high positive and negative predictive values. They concluded that in the diagnosis and management of AUB cases, the non invasive TVS should be of first choice. But hysteroscopy followed by curettage and histopathology will improve the accuracy of clinical diagnosis.

Organic pathologies which cause abnormal uterine bleeding such as endometrial polyp, myoma and endometrial hyperplasia - that are commonly not identified by blind dilatation and curettage are diagnosed readily by hysteroscopy.

Since the incidence of focal lesions in patient with AUB is high, it seems that most beneficial approach is to proceed with hysteroscopy complemented by endometrial biopsy early in the assessment of AUB. So, hysteroscopy with endometrial biopsy is the gold standard investigation for AUB.

Before hysteroscopy was available, curettage was the primary method of evaluating abnormal uterine bleeding. Brooks and Serden have revealed that approximately half of pedunculated abnormalities such as endometrial polyp were missed by curettage. It renders endometrial sampling blind and incomplete, so that the diagnostic accuracy of curettage is less than that of hysteroscopy followed by curettage biopsy. So, hysteroscopy combined with histological examination is considered better than TVS and D\&C.

\section{Bibliography}

[1]. Cicinelli E,Romana F,Anastosio psetal.,transabdominal sono hysterography ,transvaginal sonography,hysteroscopy in evaluation of organic cause of abnormal uterine bleeding.ferti/steri/2001:76:350-357

[2]. munro mg,role of hysteroscopy in diagnosis of abnormal uterine bleeding.obstet gynecol clin north am 2010:37:399-425

[3]. cooper na,khan ks et al hysteroscopy as a diagnostic tool bmj 2010:340:c1130

[4]. brooks pg serden sp hysteroscopic findings after unsuccessful dilatation and curettage for abnormal uterinne bleeding am $\mathrm{j}$ obstet gynecpol 1988:1581354-1357

[5]. loffer fd hysteroscopy with selective endometrial sampling compared with $\mathrm{d}$ and $\mathrm{c}$ for abnormal uterine bleeeding:the value of a negative hysteroscopic view obstet gynecol 19 